\title{
Analysis and Design of E-Shaped Dual-frequency Microstrip Antenna Based on CPSO Algorithm
}

\author{
AO Wei \\ Chongqing Communication College \\ Chongqing, 40035, China \\ craneaow@gmail.com \\ Chen Chun-Ming \\ Chongqing Communication College \\ Chongqing, 40035, China
}

\author{
XIANG Wan-Qin \\ Chongqing Electric Power College \\ Chongqing, 40053, China \\ TIAN WEI, ZHANG De-Bin \\ Chongqing Radiation Environment Supervision Station \\ Chongqing, 40015, China
}

\begin{abstract}
In order to solve the problem of design and optimization for E-shaped patch microstrip antenna, chaotic particle swarm optimization (CPSO) algorithm was proposed to assist the procedure. First, the E-shaped antenna's model was created, and then, the parameters of this antenna were adjusted according to the CPSO algorithm, and the procedure was repeated until the antenna's capabilities meet the requirements. The simulation results showed that, the optimized model of such antenna, was capable of dualfrequency operation in $1.8 \mathrm{GHz}$ and $2.4 \mathrm{GHz}$, and it was also capable of wide bandwidth, could meet the requirements very well.
\end{abstract}

Keywords- E-Shaped patch; microstrip antenna; CPSO

\section{INTRODUCTION}

The development of small-integrated antennas played a significant role in the progress of the rapidly expanding military and commercial communications applications. Microstrip patch antennas were widely used because of their several advantages such as light weight, low volume, low fabrication cost, and capability of dual even more frequencies operations.

For the E-shaped patch microstrip antenna, two parallel slots were incorporated to introduce a second resonant mode, resulting in a dual-band antenna, which could guarantee the channel separated and channel matched with each other. If the feed point is located at the tip of the center arm as in ${ }^{[1]}$, the second resonant mode will be introduced at a lower frequency than the fundamental resonant mode. If the feed point is moved to the base of the center arm ${ }^{[2]}$, the second resonant mode will be introduced at a higher frequency than the fundamental resonant mode.

In this paper, a wideband E-shaped microstrip antenna was designed to work in the $1.8 \sim 2.4 \mathrm{GHz}$ frequency band, and to solve the drawback of particle swarm optimization algorithm, chaotic algorithm was introduced in the optimization of the parameters of this antenna. The simulation showed that, the antenna had two resonant frequencies and wide bandwidth.

\section{GEOMETRY OF ANTENNA}

The Geometry of the E-shaped microstrip was showed in figure 1 . The geometrical parameters were the patch length $L$, the patch width $W$, the height $H$, the slot length $L_{s}$, the slot's width $W_{s}$, and the feed position $X$. And the resonant frequency and bandwidth could be optimized by adjusting these six parameters.
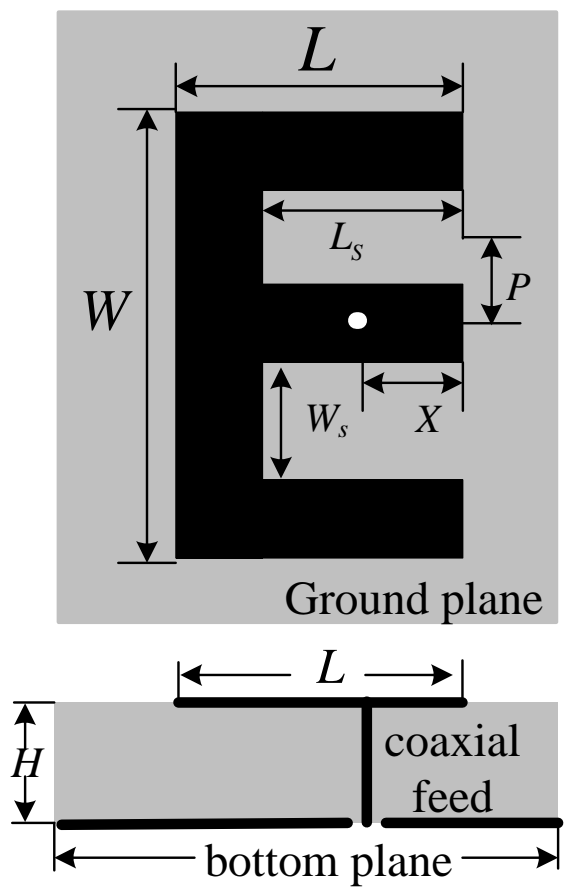

Figure 1 Configuration of E-shaped antenna

For a normal path antenna, its radiation excitation could be equivalent with a simple LC resonant circuit ${ }^{[3]}$. And for an E-shaped patch antenna, because of its slots, it could be equivalent with a dual-frequency resonant circuit, further more; its bandwidth would be expanded due to the coupling of the two channel of resonant circuit.

$\mathrm{In}^{[4]}$, the bandwidth of an E-shaped antenna was:

$$
W=\frac{c}{2 f}\left(\frac{\varepsilon_{r}+1}{2}\right)^{-1 / 2}
$$

The length was similar to $\lambda_{g} / 2, \lambda_{g}$ was the wavelength in the medium.

$$
\lambda_{g}=c / f \sqrt{\varepsilon_{r}}
$$


The length of the antenna was:

$$
L=c / 2 f \sqrt{\varepsilon_{c}}-2 \Delta L
$$

And, the effective dielectric constant was:

$$
\varepsilon_{c}=\frac{1+\varepsilon_{r}}{2}+\frac{\varepsilon_{r}-1}{2}\left(1+\frac{12 H}{W}\right)^{-1 / 2}
$$

And the $\Delta L$ was :

$$
\Delta L=0.412 H \frac{\left(\varepsilon_{c}+0.3\right)(W / H+0.264)}{\left(\varepsilon_{c}-0.258\right)(W / H+0.8)}
$$

As before, $c$ was speed of light, $f$ was operational frequency, $\varepsilon_{r}$ was relative dielectric constant, $H$ was the thickness of the dielectric plate.

Considering the size, weight, cost and other factors, the substrate plane would be as small as possible, in this paper, the size was smaller than $100 \mathrm{~mm} \times 120 \mathrm{~mm}$, and the antenna was planted on a air substrate of $15 \mathrm{~mm}$ thick. In order to designing a E-shaped with dual-frequency working among 1.8 and $2.4 \mathrm{Ghz}$, these parameters should be optimized, and they should limited as follows ${ }^{[5]}: L: 36 \sim 96 \mathrm{~mm}, \quad W: 36 \sim$ $96 \mathrm{~mm}, L_{s}: 0 \sim 96 \mathrm{~mm}, W_{s}: 0 \sim 48 \mathrm{~mm}, \quad P: 0 \sim 48 \mathrm{~mm}$, $X: 0 \sim 48 \mathrm{~mm}$. Furthermore, they should match the following inequalities:

$$
\left\{\begin{array}{l}
L_{x}<L \\
P>\frac{W_{s}}{2} \\
P+\frac{W_{S}}{2}<\frac{W}{2} \\
|x|<\frac{L}{2}
\end{array}\right.
$$

\section{CHAOS PARTICLE SWARM OPTIMIZATION ALGORITHM}

\section{A. Particle Swarm Optimization Algorithm}

Particle Swarm Optimization (PSO) algorithm was a random optimization algorithm ${ }^{[6]}$ based on swarm intelligence. At the beginning of the algorithm, the position and speed of the swarm were assigned randomly, and they were refreshed by the equations:

$\left\{\begin{array}{l}V_{i}^{(t+1)}=\omega V_{i}^{(t)}+c_{1} r_{1}\left(p_{\text {best }}^{(t)}-X_{i}^{(t)}\right)+c_{2} r_{2}\left(g_{\text {best }}^{(t)}-X_{i}^{(t)}\right) \\ X_{i}^{(t+1)}=X_{i}^{(t)}+V_{i}^{(t+1)}\end{array}\right.$

In the equations above, $V_{i}^{(t)}, V_{i}^{(t+1)}, X_{i}^{(t)}, X_{i}^{(t+1)}$ were the speed and positions in the current and the next time separately; $c_{1}, c_{2}$ were learning factors above $0 ; r_{1}, r_{2}$ were random factor between $[0,1]$; and $\omega$ was weight factor; $p_{\text {best }}^{(t)}, g_{\text {best }}^{(t)}$ were the optimization position of the particle and swarm.

The PSO would be convergent fast, but it had disadvantage that it would trapped in local minimum. In order to solve that drawback, the chaotic algorithm was introduced.

\section{B. Chaotic Optimization Algorithm}

Chaotic optimization algorithm would be convergent quickly, sensitive to initial values ${ }^{[7]}$, and easy to escape from local minimum. The chaotic sequences used in this paper were Tent sequences, whose mathematic model was:

$$
x(n+1)= \begin{cases}x(n) / 0.4 & x(n) \leq 0.4 \\ (1-x(n)) / 0.6 & x(n)>0.4\end{cases}
$$

\section{Chaotic Particle Swarm Optimization Algorithm}

The factor which affected the convergence of PSO was the diversity of particle swarm, the introduction of chaotic sequences would increase its diversity; and the $g_{\text {best }}^{(t)}$ would be modified by the Tent sequences in every iteration, which would lead a global optimization. It was called the chaotic particle swarm optimization (CPSO) algorithm, and it would be used in the optimization of E-shaped patch microstrip antenna.

\section{SimUlation AND OPTIMIZATION}

The E-shaped patch microstrip antenna would be modeled, and figure. 2 showed the model created in Ansoft HFSS.

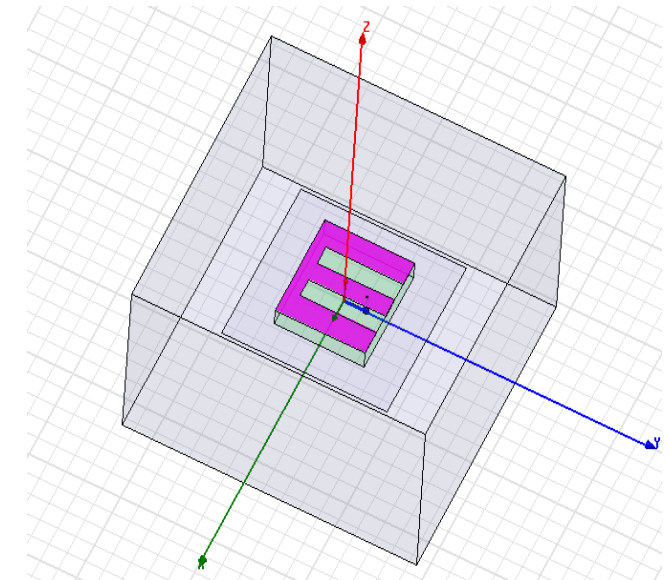

Figure 2 E-shaped patch microstrip antenna model in HFSS

The parameters of the E-shaped antenna would be optimization using the CPSO algorithm with Matlab ${ }^{[8]}$. The parameters were made as the particle of swarm, the restrict of the antennas as the fitness function, and the parameters were within (unit: mm): $L \in(36,96) ; W \in(36,96)$; $L_{s} \in(0,96) ; W_{s} \in(0,48) ; P_{s} \in(0,48) ; X \in(0,48)$. The setting of CPSO was: the swarm was 30 , the iteration numbers was 500. And the procedure was as follow:

Step1: Matlab executed the initial function, in which the particles were initialed and the parameters.

Step2: Computed the parameters of the E-shaped antennas mentioned above, using these parameters of the particles and swarm. 
Step3: Created the models of these different parameters in Matlab by calling HFSS-script, and simulated these models in HFSS.

Step4: Computed the fitness function with the $\mathrm{S}$ parameters in file tempdata.m, and optimized the particles according to the fitness.

Step5: If it was not satisfied the termination condition, go to step2.

Step6: If it was satisfied the termination condition, stored these parameters and quit the iteration.

Step7: Exit.

And table.1 compared the initialed parameters with those optimized.

TABLE 1. PARAMETERS INITIALED AND AFTER OPTIMIZATION

\begin{tabular}{lccccccc}
\hline Parameters & $L$ & $W$ & $L_{s}$ & $W_{s}$ & $P$ & $X$ & $H$ \\
\hline Initialed & 48 & 48 & 48 & 24 & 24 & 24 & 24 \\
\hline Optimized & 55 & 46 & 47 & 19 & 11 & 13 & 15 \\
\hline
\end{tabular}

Fig.3-5 showed the return loss $\left(S_{11}\right)$, the voltage standing wave ration (VSWR), and the directional diagram of the initialed model and the optimized model of E-shaped antenna.

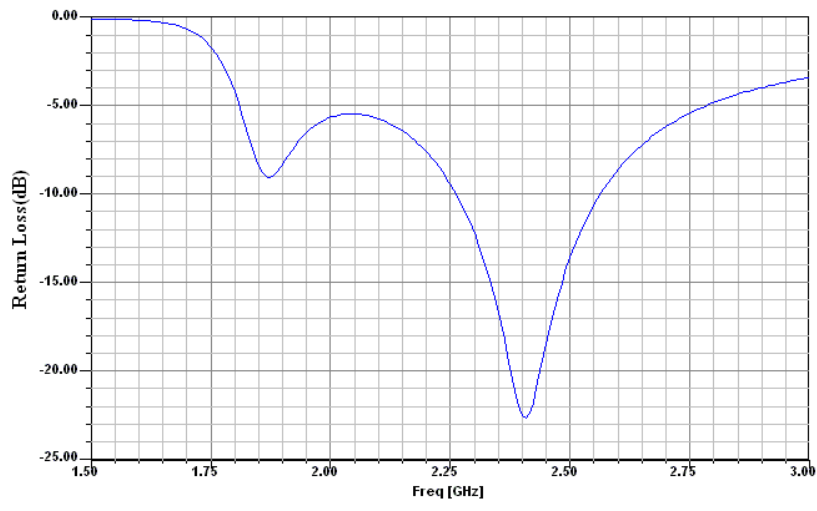

a) Return loss of the initialed antenna

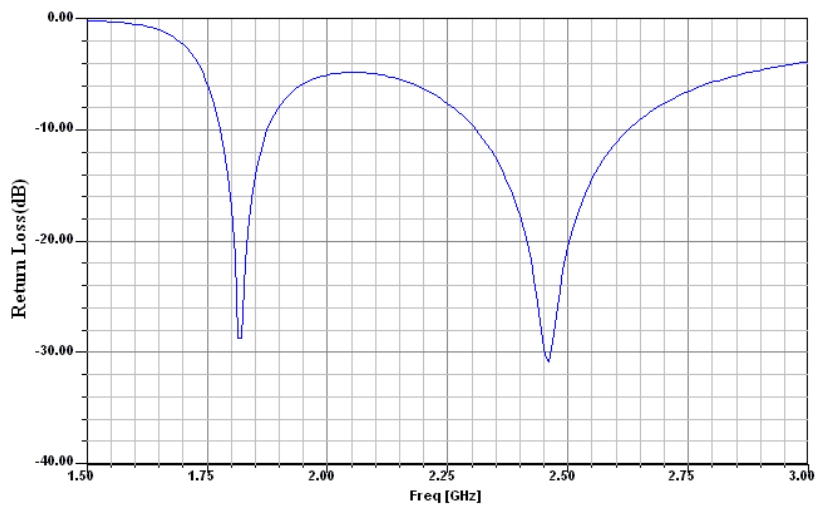

b) Return loss of the optimized antenna
Figure 3 Return loss $\left(S_{11}\right)$ of antenna

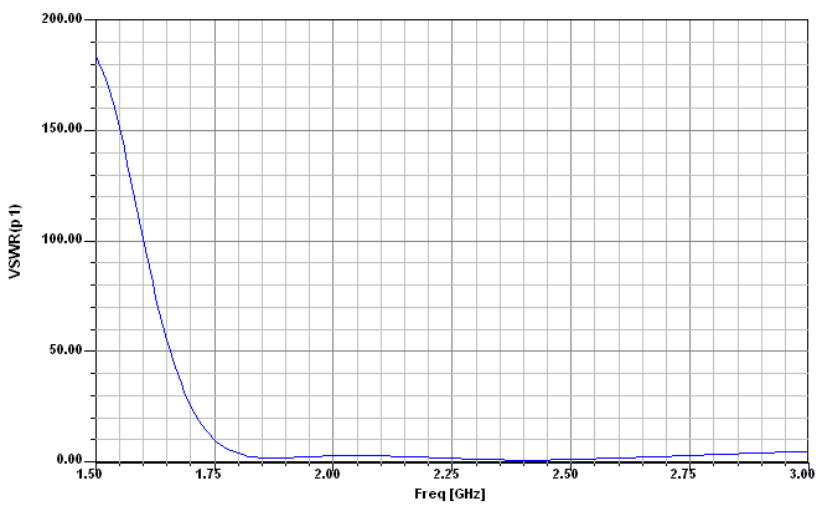

a) VSWR of the initialed antenna

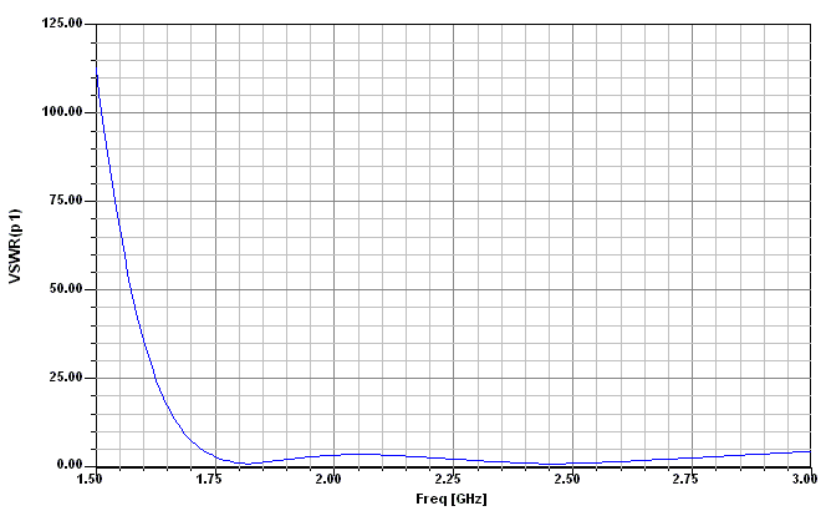

b) VSWR of the optimized antenna

Figure 4 the voltage standing wave ratio (VSWR)

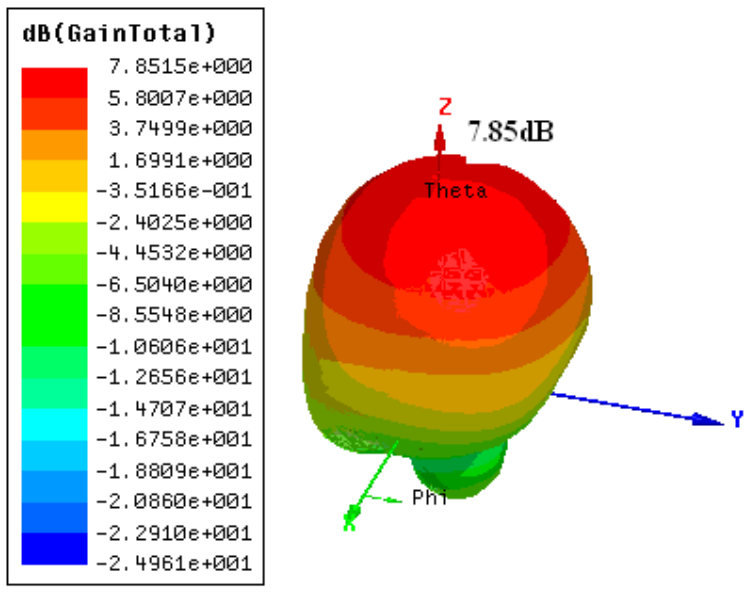

a) Directional diagram of the initialed antenna 

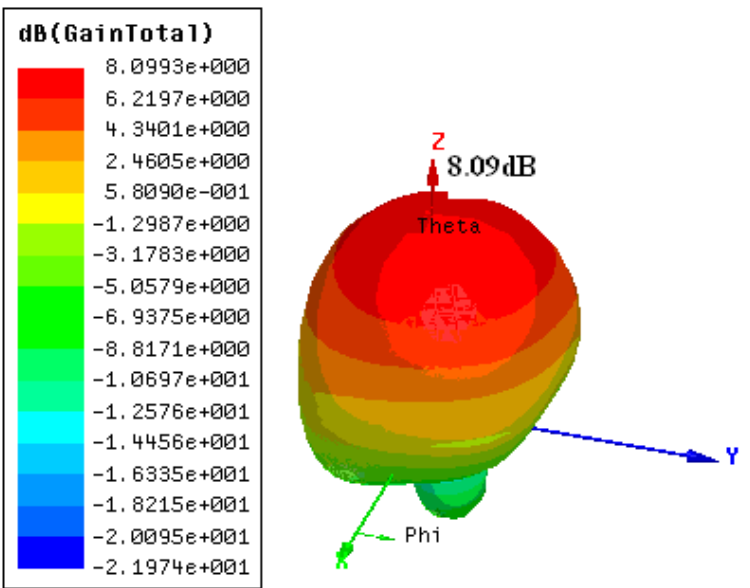

b) Directional diagram of the optimized antenna

Figure 5. Gain directional diagram in far field

From the comparison, the optimized model had advantages in both dual-frequency characteristic and bandwidth. The optimized antenna had two obvious resonant frequencies, the $S_{11}$ of both resonant frequencies were almost $-30 \mathrm{dBb}$; and the frequency band of $-10 \mathrm{~dB}$ were $1.77-1.87 \mathrm{GHz}$ and $2.3-2.63 \mathrm{GHz}$, and the bandwidth were up to $100 \mathrm{MHz}$ and $330 \mathrm{MHz}$ separately; and the voltage standing wave ratio was decreased yet; the gain in far field was increased from $7.85 \mathrm{~dB}$ to $8.09 \mathrm{~dB}$.

\section{CONCLUSION}

This paper focused on studied the E-shaped patch microstrip antenna, and proposed a method to optimize the parameters of that antenna with CPSO algorithm with Matlab and HFSS. The simulation results showed that, the optimized model had good dual-frequency characteristic, and its bandwidths expanded; and the gain increased yet. Further more, the optimized antenna could work between $1.8-2.5 \mathrm{GHz}$, and could work in the mobile communication system, such as TD-LTE.

\section{ACKNOWLEDGMENT}

This work was supported by the project of Chongqing Radiation Environment Supervision Station, 'the evaluation of the radiation of 3G base station’ (HT20120302-HB02).

\section{REFERENCES}

[1] W. L. Stutzman and G. A. Thiele, Antenna Theory and Design, $2^{\text {nd }}$ ed. New York: Wiley, 1998

[2] F. Yang, X. X. Zhang, X. Ye, and Y. Rahmat-Samii, "Wide-band Eshaped patch antennas for wireless communications," IEEE Trans. Antennas Propag., vol. 49, no. 7, pp. 1094-1100, Jul. 2001.

[3] S.Weigand, G. H. Huff, K. H. Pan, and J. T. Bernhard, "Analysis and design of single-layer rectangular u-slot microstrip patch antennas," IEEE Trans. Antennas Propag., vol. 51, no. 3, pp. 457-468, Mar. 2003.

[4] Y. Ge, K. P. Esselle, and T. S. Bird, "E-shaped patch antennas for highspeed wireless networks,” IEEE Trans. Antennas Propag., vol. 52, no. 12, pp. 3213-3219, Dec. 2004.

[5] Nanbo Jin, Yahya Rahmat-Samii. Parallel Particle Swarm Optimization and Finite-Difference Time-Domain (PSO/FDTD) Algorithm for Multiband and Wide-Band Patch Antenna Designs. IEEE Transactions on Antennas and Propagation[J], Vol.53, No.11, November 2005

[6] Shu-hui,Wang Bing-zhong.Parameter Optimization Based on GA and HFSS[J].Jounal of Electronic Science and Technology of China, 2005,Vol.3 N0.1,45-47.

[7] Liu Huaying, Lin Yue, Zhang Junshi. A hybrid particle swarm optimization based on chaos strategy to handle local convergence[J]. Computer Engineering and Applications, 2006, No.1, pp.77-79.

[8] D. Gies and Y. Rahmat-Samii, "Particle swarm optimization (PSO) for reflector antenna shaping,” in 2004 IEEE Antennas Propagat. Soc. Int. Symp. Dig., vol. 3, Jun. 2004, pp. 2289-2293. 